\title{
BUSCANDO EL DEPORTE EDUCATIVO ¿CÓMO FORMAR A LOS MAESTROS?
}

\section{Autores}

Dr. Francisco Javier Giménez Fuentes-Guerra Facultad de Educación Universidad de Huelva
Dr. José María Rodríguez López

Facultad de Educación Universidad de Huelva

\section{Resumen}

La escuela, cada vez más, ha de completar su función instructiva para asumir plenamente la función educativa o formativa. La posibilidad de integrar los contenidos de la Educación Física y especialmente los de la práctica deportiva, en la formación y educación en valores de los escolares, se ha convertido en una necesidad. Por ello, intentamos reflexionar sobre la búsqueda de ese deporte educativo que tanto nos interesa y en la forma en que podemos hacerlo asequible a los profesores y profesoras en formación..

Palabras clave: deporte educativo, valores y deporte, formación de profesores, práctica reflexiva

\section{Introducción}

El mundo globalizado de principios del nuevo siglo que nos ha tocado vivir, con su homogeneización cultural, su imparable desarrollo tecnológico, sus movimientos migratorios a gran escala, sus nuevas estructuras familiares, sus diferentes formas de acceder al trabajo, sus nuevas escalas de valores, etc. nos plantea cada día nuevos retos políticos, sociales y económicos.

En el centro de este huracán que está revolviendo hasta los cimientos de la sociedad está la escuela. Una escuela con una estructura muy parecida a la que tenía dos siglos antes, con escasos recursos $y$, sobre todo, con maestros poco preparados para hacer frente a esta gran ola de cambios. Las familias, los poderes públicos, la sociedad, en general, deja en manos de la escuela la educación que los ciudadanos necesitan. EI «eclipse de la familia» como primer elemento socializador es un hecho innegable.

«El protagonismo de la familia en la socialización primaria de los individuos atraviesa un indudable eclipse en la mayoría de los países, lo que constituye un serio problema para la escuela y los maestros.» (Sabater, 1997, 58).

La escuela, cada vez más, ha de completar su función instructiva, casi exclusiva en algunos momentos de la historia, para asumir plenamente la función educativa o formativa. Para ello necesitamos otra escuela integradora y diversa que sea capaz de desarrollar el papel protagonista que en el desarrollo de la igualdad de oportunidades le toca jugar. Que sea capaz de superar el reto de admitir la diversidad evitando las desigualdades. Una escuela flexible en espacios, tiempos, grupos y actividades. Una escuela que desarrolle un currículo centrado en problemas relevantes, que supere la división tradicional en asignaturas o áreas inconexas e integre y haga explícitos conocimientos, habilidades y sobre todos afectos y valores a través de las distintas materias.

En este sentido, la posibilidad de integrar los contenidos de la Educación Física y especialmente los de la práctica deportiva, en la formación y educación en valores de los escolares se convierte en una necesidad constante entre los profesionales de la Educación Física en los últimos años. Como ya se ha planteado en distintos trabajos (Castejón, 1995; Romero, 2001; o Giménez, 2003), lo realmente relevante no va a ser el contenido que utilicemos en la educación psicomotriz de los escolares, sino como abordemos todo el proceso de enseñanza y aprendizaje. Educar en valores significa contribuir a la función integradora del individuo, incorporando prácticas y actitudes que den paso al cumplimiento de derechos y 
responsabilidades de las personas. Los valores no se enseñan y se aprenden de la misma forma que los conocimientos y procedimientos.

La escuela sabe que no es la única responsable en este proceso, pero si que tiene una intervención importante.

La controversia que se crea alrededor de la iniciación al deporte es mucho mayor que el de cualquier otro contenido del área de Educación Física. Esto es debido fundamentalmente a la interpretación que muchas veces se le da, más cercano a la práctica deportiva de alta competición, que a una práctica educativa y formativa para alumnos de cualquier edad y nivel. Este debate de «deporte educativo si, deporte educativo, no» permite que cada día encontremos más trabajos que sitúan la práctica deportiva en las escuelas, como una actividad totalmente diferente del deporte de competición tradicional.

En este sentido, pensamos que la evolución que se ha producido en las metodologías a utilizar en las clases de Educación Física, nos obliga a plantear en la formación del profesorado de Educación Física, un deporte escolar en el que tengan cabida todos los alumnos, y en el que el fomento de distintos valores sea una de sus características más importantes. Por ello, no nos podemos quedar ahí, y pensar que la práctica deportiva educa por sí sola, sino que las programaciones didácticas deben incluir los métodos y estrategias que vamos a utilizar para aspirar a conseguirlo. Hay quien niega la necesidad de una pedagogía propia de los valores, piensan que los valores están siempre presentes en la participación en algún deporte, siendo suficiente una buena relación profesor-alumno, la relación con los compañeros, el esfuerzo, el ejemplo del profesor, etc. y puede que tengan razón, que en el currículo oculto aparezcan contenidos formativos, pero desde el punto de vista intencional de toda actividad educativa, es necesario explicitarlos y sistematizarlos.

La adquisición de valores y actitudes a través del deporte no es, pues, el resultado de una información pasiva ni de la comprensión que de la misma extrae el sujeto, sino algo más complejo que tiene que ver con componentes de la personalidad y sus formas de expresión a través de conductas y comportamientos.

Por todo esto, intentamos en este artículo reflexionar sobre algunas cuestiones que nos parecen de importancia en la búsqueda de ese deporte educativo que tanto nos interesa y en la forma en que podemos hacerlo asequible a los profesores y profesoras en formación. Planteamos en primer lugar nuestra concepción de la iniciación deportiva. Seguimos sintetizando un currículo básico que nos sirva de marco o referencia en la programación de cualquier unidad didáctica relacionada con la enseñanza del deporte. Exponemos algunas reflexiones personales sobre algunos elementos que nos parecen claves y referentes claros de cómo podría plantearse el proceso educativo. Analizamos los principios didácticos que debe seguir la formación del profesorado dirigido a la reflexión.

\section{Concepto de} iniciaciòn deportiva

Hemos estudiado el concepto de iniciación deportiva de autores como Blázquez $(1986,1995)$, Castejón (1995), Romero (2001), Antón (1990), Pintor (1989) u otros; y a partir de ellas proponemos nuestra propia definición, planteando ésta siempre como un contenido motriz con unos valores educativos importantes: etapa en la que una o varias personas comienzan el aprendizaje, de forma lúdica, de las habilidades genéricas y específicas necesarias para iniciar la práctica de uno o varios deportes, desarrollando su condición física de forma saludable, aprendiendo progresivamente las reglas básicas de la modalidad deportiva que se lleve a cabo, favoreciendo una actitud positiva hacia la práctica de actividad física, y promoviendo una verdadera educación en valores entre el alumnado.

Intentamos con esta definición de jar constancia de la importancia de buscar a través de la enseñanza del deporte desde una óptica educativa no sólo aprendizajes técnicos y físicos como ha sido tradicional, sino dar mayor importancia si cabe al proceso en el que se desarrolla. Por ello, nos parece imprescindible que el aprendizaje sea atractivo y motivante para todos los alumnos sin excepciones, a la vez que se fomenten hábitos de práctica y se promuevan valores educativos. Para conseguirlo, lógicamente será necesario que el profesor o el entrenador diseñe y oriente su enseñanza en este sentido, y se olvide de los valores más tradicionales y típicos del deporte de alta competición: selección de los mejores, competición como fin, falta de socialización, importancia del resultado, etc

\section{Curriculo básico}

En estrecha vinculación con el resto de contenidos de Educación Física que se llevan a cabo en las clases de Educación Primaria, exponemos a continuación los elementos curriculares básicos que habría que tener en cuenta en la programación de iniciación deportiva (Giménez, 2003). 


\subsection{Finalidades educativas}

Partiendo de los objetivos que Antón (1990) enuncia en la iniciación al balonmano, Giménez y Sáenz-López (2004) añaden algún otro en la iniciación al baloncesto que nos parece interesante destacar y que se pueden extrapolar a la iniciación deportiva en general:

Mejorar la condición física y la salud de los alumnos. Este objetivo va a conllevar, entre otras cosas, que trabajemos las cualidades físicas adecuadas a la edad, que los contenidos técnicotácticos sean idóneos, que las actividades se realicen de forma segura evitando accidentes innecesarios, que fomentemos hábitos higiénicos o que trabajemos de forma motivante. La mejora de la salud la extenderemos a los ámbitos físico, psíquico y social de los alumnos. y adversarios, y fomentar la cooperación entre los compañeros para un fin común: disfrutar jugando.

Conseguir hábitos de práctica deportiva. Buscamos conseguir hábitos saludables de práctica de actividad física de forma continuada entre todos los alumnos, independiente del nivel que vayan alcanzando. Buscaremos que disfruten con el deporte y que en el futuro lo practiquen y lleven una vida menos sedentaria.

Contribuir a la educación en valores del alumnado. Debemos orientar nuestra enseñanza de forma que consigamos fomentar valores tan importantes como la libertad, la solidaridad, la ecología, la paz, la integración y el respeto de todos los alumnos o la autonomía.

Además de estos objetivos generales que proponemos, cada profesor se planteará otros específicos de cada una de las modalidades deportivas desarrolladas, y contextualizadas en su

Cualidades físicas: coordinación, resistencia cardiovascular, fuerza y resistencia muscular, flexibilidad

Contenidos técnico-tácticos: utilización de habilidades genéricas en un deporte en concreto, habilidades específicas más básicas e importantes

Cualidades psicológicas conocimiento de nuestras posibilidades y limitaciones, espíritu de superación, desarrollo del autocontrol y la responsabilidad, autovaloración y autoestima, motivación

Valores educativos: hábitos saludables, autonomía, creatividad, igualdad, libertad, solidaridad, ecología

Cuadro 1. - Contenidos a desarrollar en la enseñanza del deporte educativo.

Trabajar los contenidos técnico-tácticos básicos. Buscaremos sobre todo que los alumnos se familiaricen con el deporte y que conozcan los principales elementos y medios técnico-tácticos que lo componen. Trataremos de evitar una especialización demasiado temprana.

Enseñar las reglas básicas del deporte. La enseñanza de las reglas debe ser progresiva. En el primer contacto con el deporte debemos enseñar de forma global lo imprescindible para poder jugar. sin importarnos demasiado que los chicos cometan diferentes violaciones al reglamento. Al igual que los contenidos técnico-tácticos, las reglas también deben ser programas en el aprendizaje de los alumnos.

Mejorar la socialización y el trabajo en equipo. Este objetivo supone desarrollar o me jorar aspectos sociales como el respeto entre compañeros lugar y centro de trabajo: aumentar el número de participantes, conocer nuevas prácticas deportivas, permitir la posibilidad de ocupar el tiempo libre, entender el deporte como un contenido educativo de interés, diferenciar el deporte educativo del deporte de alta competición, fomentar una práctica deportiva para todos, mejorar la condición motriz de los alumnos, etc.

\subsection{Contenidos}

Los contenidos que llevamos a cabo dentro de la iniciación deportiva deben buscar un desarrollo integral de los alumnos. Desde este punto de vista habrá que desarrollar cada práctica incidiendo tanto en el ámbito físico como psicosocial de cada alumno: 
Queda claro, por tanto, que debemos tener siempre presente el desarrollo de contenidos que mejoren las cualidades físicas y motrices, a la vez que se mejoren determinadas cualidades psicológicas y se fomenten valores educativos que colaborarán en la formación de todo el alumnado.

\subsection{Metodología}

Pensamos que la enseñanza del deporte ha cambiado bastante en los últimos años. En la didáctica de la iniciación deportiva hemos pasado a utilizar nuevos métodos de enseñanza alternativos y constructivistas, donde se tiene mucho más en cuenta al alumno, sus capacidades, y el contexto donde se mueve. Hemos pasado de valorar por encima de todo los resultados, a valorar los aspectos más educativos que se dan en este proceso. A pesar de ello, queda mucho camino por andar.

En este proceso de exploración aquellos profesores y profesoras que buscan una práctica deportiva formativa y educativa deberían optar por cumplir las siguientes orientaciones didácticas:

- Utilizar métodos de enseñanza específicos para la enseñanza del deporte con niños, claramente diferentes a la metodología que se utiliza con adultos y en la alta competición.

- Adaptar el deporte al niño, y no el niño al deporte. Se adaptarán reglas y materiales, la actuación del profesor/entrenador será diferente, los objetivos o finalidades se adaptarán a cada. contexto particular de actuación.

- No adelantar la edad de inicio que la mayoría de los autores sitúa en torno a los 9 años.

- Menor importancia en el desarrollo tradicional de la técnica y de la competición, en beneficio de la táctica y de la participación.

- No seleccionar a los jugadores, sino integración y participación de todos sin excepciones.

- Práctica variada de deportes y actividades. Tener en cuenta los intereses de los alumnos.
- Proceso de formación a largo plazo, evitando una especialización demasiado temprana.

- Formación integral del alumnado. Para ello programaremos en cada sesión objetivos no sólo físicos y técnicos, sino también finalidades relacionadas con el fomento de valores educativos. El deporte por sí mismo no genera valores educativos como tantas veces escuchamos, sino que hay que orientar nuestra enseñanza en ese sentido para conseguir este importante objetivo.

- Las sesiones de enseñanza se llevarán a cabo a través de actividades lúdicas donde el alumno tenga siempre una implicación tanto motriz como cognitiva.

Búsqueda del consenso con los alumnos sobre las actividades de aprendizaje.

Participación de los alumnos en la definición de las normas para la realización de las tareas y de convivencia en la clase.

Participación de los alumnos en la aplicación de las normas y en la resolución de conflictos.

Utilización de una competición diferente, donde ésta se utilice como medio de motivación y disfrute en la puesta en práctica de las actividades.

Etc.

\subsection{Evaluación}

Como ya hemos comentado la valoración del deporte debe seguir cambiando. Pensamos que la iniciación deportiva puede y debe ser evaluada como un contenido más de la Educación Física. Debido a ello, será un proceso de valoración continua y global, no sólo del alumnado, donde tendremos en cuenta aspectos actitudinales y procedimentales, y también aunque en menor medida, aspectos conceptuales. Se tendrán en cuenta tanto las características específicas de cada alumno como el contexto general en el que se desarrolla el proceso educativo, primando sobre

\begin{tabular}{|l|l|l||}
\hline \multicolumn{1}{|c|}{ ÁMBITOS } & \multicolumn{1}{|c|}{ TÉCNICAS } & \multicolumn{1}{c|}{ INSTRUMENTOS } \\
\hline Afectivo & - Observación & - Hojas de registro \\
\cline { 1 - 2 } Motriz & - Observación & - Cuaderno del alumno \\
& - Pruebas motrices & - Cuestionarios \\
\multirow{2}{*}{ Cognitivo } & - Información directa & - Entrevistas \\
& - Pruebas Tradicionales & - Pruebas de ensayo \\
& & - Pruebas objetivas \\
\hline
\end{tabular}

Cuadro 2. - Propuesta de técnicas e instrumentos de evaluación en la iniciación deportiva. 
todo el esfuerzo por encima de los resultados. Por tanto, se tratará de que valoremos a los alumnos en función de sus capacidades individuales, y atendamos no sólo a cuestiones relacionadas con su aptitud física, sino teniendo en cuenta su actitud hacia la asignatura y los contenidos que en ella tratamos. Se incluirá la autoevaluación como un componente importante en el proceso.

Para valorar los distintos ámbitos que planteamos, será necesario utilizar diferentes instrumentos de evaluación que nos ayuden en este complicado proceso. En el siguiente cuadro exponemos una serie de herramientas que nos pueden ser de utilidad, recordando siempre que deben ser adaptadas a nuestro contexto particular, añadiendo o suprimiendo las que creamos convenientes:

\section{Formación de prácticos reflesivos}

Partimos del supuesto de la imposibilidad de preparar a los profesores para todas las circunstancias que a lo largo de su vida profesional se le van a presentar. Las situaciones de enseñanzaaprendizaje son impredecibles, únicas e irrepetibles. Por ello hemos de preparar a los profesores para que de una manera responsable, abierta y creativa, afronten de manera reflexivay profesional los problemas que a lo largo de su carrera se le vayan presentando.

Se trata, por tanto, de conseguir que desarrollen una práctica profesional reflexiva (Järvinen, 1989, 4) a través de la cual y mediante una continua reflexión crítica sobre sus percepciones, pensamientos y acciones, intenten desarrollar y cambiar su propia práctica y el contexto social en que la llevan a cabo.

Pollard y Tann (1989) identifican cuatro características esenciales de la enseñanza reflexiva:
1. Implica una preocupación activa sobre propósitos y consecuencias, así como sobre eficacia técnica y significativa.

2. Combina habilidades de indagación y desarrollo con actitudes de apertura de pensamiento, responsabilidad y entusiasmo (actitudes que ya enunciara Dewey como favorables)

3. Es aplicada en un proceso circular y espiral, en el que los profesores observan, evalúan y revisan su propia práctica.

4. Está basada sobre los juicios de los profesores, informados especialmente por la autorreflexión y por las intuiciones de las distintas disciplinas educativas.

Por lo tanto un profesor reflexivo delibera continuamente sobre los objetivos que se plantea, las actividades y resultados de su enseñanza, y considera a corto y largo plazo las repercusiones que pueden tener sobre sus alumnos.

Como consecuencia de ello, la preparación de profesionales reflexivos requiere tener en cuenta una serie de principios didácticos:

Un enfoque integrador de los distintos contenidos con los elementos psicopedagógicos y didácticos necesarios para desarrollar su enseñanza. En el caso que nos ocupa, la iniciación al deporte, es necesario integrar los valores de manera intencionada y consciente en cada uno de los temas, reflexionando sobre la relación que tienen cada uno de los contenidos y habilidades que queremos que aprendan los futuros maestros con los valores que implícitamente pueden aparecer. Esto significa ofrecer un enfoque holístico y complejo del aprendizaje, en el que los futuros profesores reflexionan sobre el valor educativo de sus propias actividades de aprendizaje.

-Isomorfismo entre la manera en que se educa a los profesores y la forma en que estos han de educar a sus alumnos. Educar en valores significa aprender los valores apreciándolos, es decir, incorporando prácticas y actitudes que permitan

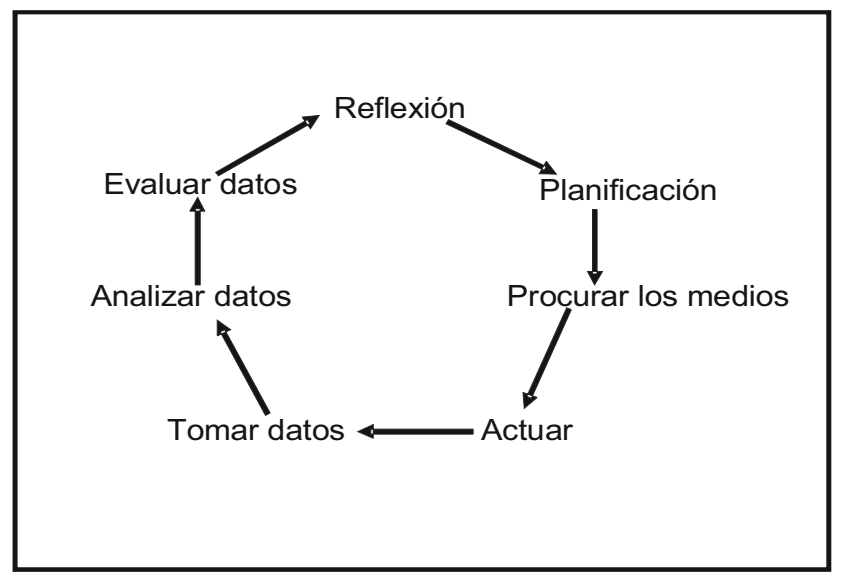

Gráfico 1. - El Proceso de Enseñanza Reflexiva (Pollard, A. y Tann, B., 1989, 8). 
experimentar derechos y responsabilidades de igual manera como les proponemos que lo hagan con sus alumnos. Es un proceso a largo plazo (en realidad dura toda la vida), siempre provisional, que va estar condicionado por los cambios sociales que se producen y las condiciones materiales y naturales en las que se desarrolla la vida de los individuos.

- Alternancia entre períodos de teoría y práctica que podemos compaginar con la estructura de la propia asignatura procurando que las clases se desarrollen de forma teórico-práctica. Buscaremos, sobre todo, un espacio de reflexión activo de manera que se provoque la deseada integración dinámica y equilibrada de esos dos mundos tradicionalmente separados. Se tratará de incluir a través de las propias clases prácticas conceptos relacionados con el aprendizaje educativo del deporte, de forma que facilitemos una práctica crítica, respetuosa y autónoma.

- Formación activa en procesos de indagaciónreflexión por los que los futuros profesores podrán seguir después de manera continua desarrollando su propio conocimiento profesional y sus propias teorías. Se trata de hacerles pasar de consumidores del conocimiento profesional que elaboran otros a producir o colaborar en la producción del cuerpo científico que les puede ayudar a interpretar $y$ entender lo que sucede en sus clases.

-Evaluación basada también en criterios de desempeño, no exclusivamente en criterios de conocimientos. La forma en que se produce la evaluación tiene tal fuerza sobre los procesos de enseñanza-aprendizaje que muchas veces marca el camino de lo que los alumnos quieren/deben aprender. Por ello, es necesario poner en valor las competencias de los profesores en formación en el desempeño de todas las tareas docentes. De las más conocidas que se desarrollan en el aula y frente a sus alumnos, y de las que se desarrollan fuera del aula: planificando, corrigiendo trabajos, coordinándose con otros compañeros para la realización de trabajos de grupo, etc. Las nuevas perspectivas metodológicas en la enseñanza del deporte dejan claro que el propio alumno y su proceso de aprendizaje es lo verdaderamente importante. Por ello, planteamos como contenidos principales los actitudinales frente a los conceptos y las aptitudes como ha sido más tradicional.

\section{Bibliografía}

AA.VV. (1988). Diccionario de las Ciencias de la Educación. Madrid: Aula Santillana.

Antón, J. (1990). Balonmano, fundamentos y etapas de aprendizaje. Madrid: Gymnos.

Blázquez, D. (1986). Iniciación a los deportes de equipo. Barcelona: Martínez Roca.
Blázquez, D. (1995b). A modo de introducción. En Blázquez, D. (Dir). La iniciación deportivay el deporte escolar. Barcelona: INDE.

Blázquez, D. (1995c). Métodos de enseñanza de la práctica deportiva. En Blázquez, D. (Dir). La iniciación deportiva y el deporte escolar. Barcelona: INDE.

Castejón, F. J. (1995). Fundamentos de iniciación deportiva y actividades físicas organizadas. Madrid: Dykinsón.

Contreras, O. R.; De La Torre, E. y Velázquez, R. (2001). Iniciación Deportiva. Madrid: Sintesis.

Devís, J. y Peiró, C. (1992). Orientaciones para el desarrollo de una propuesta de cambio en la enseñanza de los juegos deportivos. En Devís, J.; Peiró, C. Nuevas perspectivas curriculares en E.F.: la salud y los juegos modificados. Barcelona: INDE.

Dewey, J. (1989). Cómo pensamos. Madrid: Paidós.

Díaz, J. (1998). El currículum de la E. F. en la reforma educativa. Barcelona: INDE.

Giménez, F. J. (2000). Fundamentos básicos de la iniciación deportiva en la escuela. Sevilla: Wanceulen.

Giménez, F. J. (2003). El deporte en el marco de la Educación Física. Sevilla: Wanceulen.

Giménez, F. J.y Sáenz-López, P. (2004). Aspectos teóricos y prácticos de la iniciación al baloncesto. Sevilla: Wanceulen.

Jarvinen, M. (1989). Development of reflection during teacher education. Paper presented at the Third European Conference for research on learning and instruction.

Méndez, A. (1999). Modelos de enseñanza deportiva. Análisis de dos décadas de investigación. Lecturas de E. F. y deportes, $n^{\circ} 13$.

Pintor, D. (1989). Objetivos y contenidos de la iniciación deportiva. En ANTÓN, J. (Coord). Entrenamiento deportivo en la edad escolar. Málaga: Unisport.

Pollard, A.; Tann, S. (1989). Reflective Teaching in the primary school. A Handbook for the classroom. London: Cassell.

Rodríguez, J. M. (1995). Formación de profesores y prácticas de enseñanza. Un estudio de caso. Huelva: Servicio de Publicaciones de la Universidad de Huelva.

Rodríguez, J. M. (2001). Bases y estrategias de formación permanente del profesorado. Huelva: Hergué.

Romero, S. (2001). Formación deportiva: nuevos retos en educación. Sevilla: Universidad de Sevilla.

Ruiz, F.: García, A. y Casimiro, A. (2001). La iniciación deportiva basada en los deportes colectivos. Madrid: Gymnos.

Savater, F. (1997). El valor de educar. Barcelona: Ariel. 